\title{
Concept de poste roulier flottant
}

Jean-Michel SEVIN

Service E.T.I., Port Autonome de Nantes / Saint-Nazaire

Le second poste roulier de Montoir-de-Bretagne entre en service à la fin du premier trimestre 1996. Cet équipement d'envergure et d'une technologie originale, permettra de doubler la capacité d'accueil des navires Roll-on Roll-off du Port Autonome de Nantes-Saint-Nazaire.

Contrairement au poste roulier $n^{\circ} 1$, classique passerelle sur vérins, le nouveau poste implanté dans l'estuaire de la Loire est une structure flottante sur deux coques. Ce prototype a été imaginé, mis au point et testé par les bureaux d'études Technitas, Normandie-Ingenierie et Carehna pour le compte des Constructions Métalliques Paimboeuf.

Implanté dans l'estuaire de la Loire, à $100 \mathrm{~m}$ de la rive, il est soumis à des conditions océanographiques qui ont nécessité des études de comportement à l'aide de modèles tant mathématiques que physiques. La spécificité technique de cette opération a nécessité de la part de l'équipe de conception recherche et rigueur, pour concevoir un ouvrage qui allie des qualités d'adaptabilité et de souplesse d'utilisation. 


\section{1 - PRÉSENTATION DE L'OPERATION}

\section{1 - Définition des besoins}

Jusqu'à cette année le terminal roulier de Montoir était desservi par un seul poste roulier mis en service en 1976. Il assurait la réception des navires à rampe axiale de 9000 te déplacement maximum.

Cet ouvrage supportait à lui seul, à raison de 5 escales de navires par semaine, plus de $35 \%$ du tonnage de marchandises diverses traité par le Port Autonome (627 000 t/ $7000000 \mathrm{t}$ en 1993) et $55 \%$ du trafic de ligne.

Le poste actuel était donc saturé et son exploitation à la merci d'immobilisations par incidents ou avaries susceptibles de freiner le développement des lignes régulières et allait à l'encontre de la volonté affirmée du Port de renforcer le trafic de marchandises diverses sur le terminal de Montoir.

La construction d'un deuxième poste roulier s'est donc révélé comme l'une des priorités d'aménagement de la Zone Industrielle Portuaire de Montoir. En permettant de doubler la capacité d'accueil des navires rouliers, cet ouvrage fiabilise le trafic existant et favorise l'arrivée et le développement de nouvelles lignes maritimes.

L'ouvrage est conçu pour la réception des navires rouliers à rampe axiale ou latérale oblique de $15000 \mathrm{t}$ de port en lourd maximum, de $180 \mathrm{~m}$ de longueur et de $9 \mathrm{~m}$ de tirant d'eau.

Il comporte pour ce qui concerne les superstructures métalliques un poste de transbordement constitué par un ponton flottant guidé et une passerelle carrossable d'accès au ponton.

Les ouvrages d'infrastructure permettant l'accostage et l'amarrage des navires sont constitués par quatre ducs d'Albe d'accostage et cinq ducs d'Albe d'amarrage desservis par des passerelles piétons. Ils comprennent également les ouvrages de guidage du ponton et les appuis des passerelles d'accès.

Ces ouvrages dinfrastructure ne sont pas concernés par la présente présentation. 


\section{2 - Obiet de la consultation du port}

La commande du port auprès des Ateliers et Chantiers de Paimboeuf (ACP) devenu depuis Construction Métallique Paimboeuf (CMP) avait pour objet l'étude, la fourniture, le transport, le montage sur site et les essais du ponton flottant guidé et de sa passerelle d'accès devant constituer les superstructures métalliques du poste roulier projeté.

Les ouvrages réalisés selon le plan masse annexe 1 comprennent :

- un ponton flottant en acier de $60 \mathrm{~m} \times 25 \mathrm{~m} \times 3,60 \mathrm{~m}$ équipé de ses colliers de guidage et constitué par un ponton principal de $45 \mathrm{~m} \times 25 \mathrm{~m}$ et d'un ponton additionnel amovible de $15 \mathrm{~m} \times 25 \mathrm{~m}$ selon la configuration du schéma annexe 2.

- une passerelle carrossable en acier de $11,20 \mathrm{~m}$ de largeur pour l'accès au ponton constituée de 2 éléments: une passerelle fixe de $55 \mathrm{~m}$ de longueur et une passerelle mobile également de $55 \mathrm{~m}$ selon plan de principe annexe 3.

- les équipements, appareillages et dispositifs mécaniques, hydrauliques et électriques du ponton et de la passerelle, nécessaires à l'exploitation des ouvrages.

Cette opération a été réalisée dans le cadre d'un marché public industriel passé. En raison de la spécificité du projet à réaliser en site maritime (passerelle vérinée ou ponton flottant) et de la nécessité de traiter avec des entreprises possédant des structures d'études, des compétences et des"références affirmées pour ce type d'ouvrage, la procédure d'appel d'offres restreint s'est imposée : appel d'offres lancé au niveau de la Communauté Européenne.

La consultation portait sur l'étude et la définition des superstructures métalliques sur la base des plans-guides et spécifications techniques imposées dans le D.C.E., ces ouvrages pouvant être, suivant le choix final du maitre d'ouvrage au vu des résultats de l'appel d'offres : hydrauliques;

- soit une passerelle métallique articulée actionnée par vérins

- soit un ponton flottant guidé avec passerelle d'accès métallique. 


\section{3 - L'intérêt d'une struccure flottante}

L'analyse a conduit à retenir la solution "ponton flottant" de préférence à la solution "passerelle vérinée". Les avantages de cette configuration sont apparus notamment déterminants pour :

- garantir une plus grande fiabilité de l'installation par sa moindre sophistication qui implique un fonctionnement mécanique moins délicat. Son état statique est par nature moins aléatoire que celle d'une structure vérinée, même si le premier poste roulier équipé ainsi n'a jamais été arrêté pour incident technique,

- réduire les coûts d'exploitation et de maintenance,

- accueillir des navires de servitude pour lesquels un poste indépendant était projeté à court terme,

- s'adapter à d'autres typologies de navires,

- évoluer, grâce à la possibilité d'utilisation symétrique du ponton (amont-aval) vers un terminal à 2 postes d'accostage,

- être éventuellement transféré sur un autre site.

Enfin, la solution ponton apporte une nouvelle polyvalence complémentaire au poste roulier $\mathrm{n}^{\circ} 1$ et constitue un indéniable atout commercial pour mieux répondre aux évolutions du trafic roulier prévisible. 


\section{2 - CONCEPTION DE L'OUVRAGE ELOTTANT}

\section{1-Généralités}

La conception du poste roulier $\mathrm{n}^{\circ} 2$ à Montoir développée par la Société Technitas devait répondre à diverses exigences en terme de performances opérationnelles dans un site en estuaire exposé à l'action de l'environnement océanique.

L'option fondamentale du port pour une structure flottante guidée sur pieux a conduit le concepteur à rechercher un projet de flotteur "compatible" c'est-à-dire capable :

- d'accueillir des navires jusqu'à $27 \mathrm{~m}$ de largeur et dotés de rampes larges $(15 \mathrm{~m})$ droites ou obliques,

- de supporter par le travers une passerelle de $56 \mathrm{~m}$ de longueur (pour sa partie mobile) et de 260 t environ, $120 t$

- de permettre le transit de charges roulantes, jusqu'au convoi de

- d'assurer une exploitation normale sous un état de mer de $1 \mathrm{~m}$ de hauteur significative (2,00 m crête-creux).

\section{2 - L'environnement}

- Le dispositif est distant de la berge de $100 \mathrm{~m}$ environ.

- La profondeur d'eau minimale est voisine de $10 \mathrm{~m}$, le régime des marées conduit à une profondeur d'eau maximum d'environ $16,50 \mathrm{~m}$.

- L'ouvrage doit être opérationnel sous un courant de 6 noeuds au jusant et 5 noeuds au flot.

- La houle exceptionnelle servant de base au dimensionnement de l'ouvrage s'élève à $3,90 \mathrm{~m}$ de crête-creux extrême pour une période associée de $6 \mathrm{~s}$. Il peut être noté que les tempêtes de SW, les plus dommageables sur l'estuaire de la Loire produisent des houles de périodes significatives comprises entre 4 et $5 \mathrm{~s}$.

- L'incidence de la direction de propagation de la houle est de $30^{\circ}$ par rapport à l'axe de l'ouvrage.

- Le vent exceptionnel (règle NV 84) s'élève à $160 \mathrm{~km} / \mathrm{h}$. 


\section{3 - Caractéristiques principales}

\subsection{1 - Le ponton}

Le ponton devrait disposer d'une surface de roulage suffisante pour assurer un confort de déplacement des véhicules, ceci pour tous les navires spécifiés par le Port.

Une plateforme de $60 \mathrm{~m}$ de longueur par $25 \mathrm{~m}$ de largeur permet une bonne circulation des véhicules tout en réservant une quarantaine de mètres pour le positionnement d'un semi-remorque perpendiculairement à l'axe de son arrivée sur le ponton par la passerelle.

Le franc-bord de l'ouvrage est délibérément limité à environ $1,70 \mathrm{~m}$ afin d'obtenir une pente acceptable avec un navire ayant un seuil de $1 \mathrm{~m}$ seulement de franc-bord et une rampe de $6 \mathrm{~m}$ de longueur.

Le choix d'une structure de type semi-submersible étant a priori écarté en raison des variations de tirant d'eau gite et assiette au passage des charges, l'alternative essentielle résidait alors entre un flotteur prismatique simple ou un catamaran. suivantes:

Le catamaran a été retenu notamment pour les raisons

- réduction du poids de coque métallique à surface de pont égale,

- optimisation des volumes de ballast,

- excellente stabilité hydrostatique transversale optimisant la gite en opération.

\subsection{2 - Le problème de la rampe oblique}

Une telle structure du pont rectangulaire restait inadaptée aux opérations avec navires dotés de rampes obliques. Pour remplir cette fonctionnalité, le ponton de $60 \mathrm{~m} \times 25 \mathrm{~m}$ est constitué de 2 parties:

- un ponton principal de $45 \mathrm{~m}$ de longueur,

- un ponton amovible de $15 \mathrm{~m}$ de longueur.

Le ponton amovible est situé côté aval. 
Les opérations de connection/déconnection sont assurées à flot par mer calme; la liaison est réalisée par 4 broches vérinées, verrouillage 1/4 de tour (voir schéma annexe 4 .)

Lors de l'accueil d'un navire à rampe oblique, le tronçon amovible est amené en abord par le travers du ponton principal, offrant ainsi une largeur supplémentaire de $15 \mathrm{~m}$ en bout de navire, soit $40 \mathrm{~m}$ sur une profondeur de $25 \mathrm{~m}$.

L'effet de coin entre ces structures à angle droit sur la houle étant susceptible de provoquer des embruns, un biais a été ajouté, solitaire du ponton amovible. Ce biais ajoute une aisance supplémentaire aux opérations de roulage.

\subsection{3 - Le guidage}

L'ouvrage flottant est soumis à l'action de la marée et des vagues. Le dispositif de guidage du ponton devait tenir compte de cet environnement relativement hostile en situation de tempête et assure les fonctionnalités suivantes :

- coulissage vertical pour absorber l'effet de marée et le pilonnement dû à la houle ;

- liberté en tangage et roulis à l'intérieur de certaines limites afin de restreindre les mouvements relatifs horizontaux navire-ponton d'une part, passerelle-ponton d'autre part.

Les pieux de guidage monotubulaires à inertie variable ont un diamètre de $2,40 \mathrm{~m}$, et sont distants de $38 \mathrm{~m}$ en abord du ponton principal.

Les colliers de guidage sont constitués de rotules et dotés de patins plastiques. Les patins assurent à la fois la fonction coulissage le long du pieu et la fonction rotation pour les mouvements de roulis-tangage (voir croquis annexe 5).

La rotule aval est montée sur chariot coulissant afin d'autoriser le mouvement de tangage.

\subsection{4 - L'appui passerelle}

La passerelle mobile de $56 \mathrm{~m}$ de longueur s'appuie sur le ponton principal à $17,50 \mathrm{~m}$ de son extrémité amont. 
L'appui est assuré par un système de boogies qui autorise les déplacements relatifs dans le plan du pont du ponton.

\section{4 - La modélisation mécanique}

\subsection{1 - Efforts hydrodynamiques}

L'action des éléments sur le ponton peut être sériée en 2 composantes :

- Efforts permanents ou quasi-permanents:

Il s'agit de l'action du courant, du vent et de la houle pour ce qui concerne sa composante de dérive moyenne sur mer irrégulière.

\section{Efforts dits "haute fréquence" :}

Il s'agit des efforts sur houle agissant à la fréquence de la houle.

La méthode retenue a consisté à traiter séparément ces deux phénomènes puis à les cumuler. Cette approche est admise ici car l'action "basse fréquence" des vagues n'excite pas les mouvements horizontaux du ponton, leurs périodes propres étant très basses.

\subsection{2 - Le problème mécanique}

Outre les composantes hydrodynamiques, le flotteur est soumis :

- aux efforts d'inertie de masse propre,

- aux efforts de rappel hydrostatique,

- aux efforts de liaison avec les pieux.

Cette dernière composante est approché par une force de rappel proportionnelle à la rigidité $\mathrm{K}$ du pieu. Cette rigidité est supposée constante dans toutes les directions horizontales et ne dépendra que de la profondeur d'eau. 
2.5 - Principales domnées

\subsection{1 - Ponton(s)}

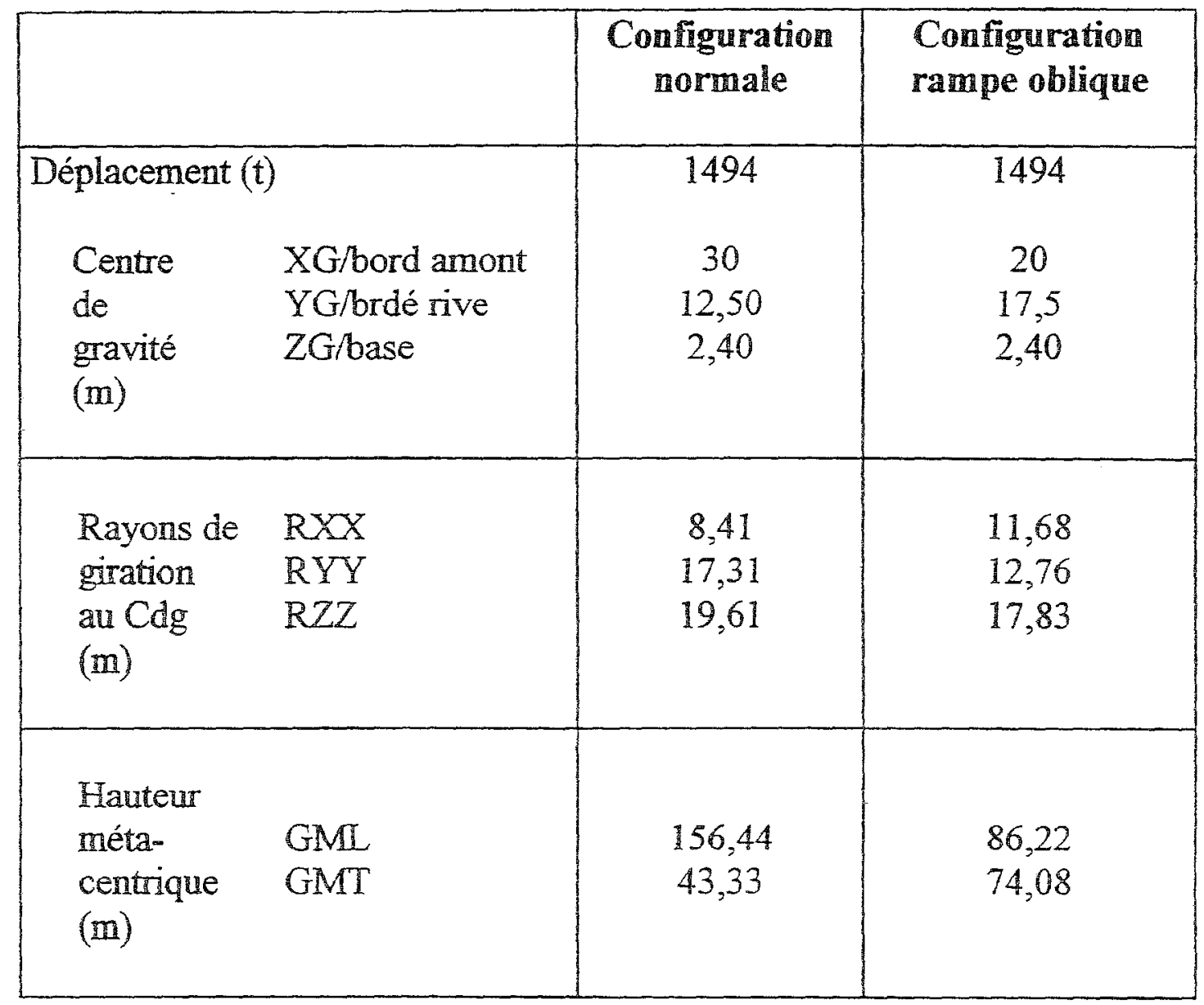

2.5.2- Passerelle

Déplacement : $260 \mathrm{t}$

Centre de gravité

Longitudinal/axe chamière quai

$16,45 \mathrm{~m}$

Transversal/axe passerelle

$1,73 \mathrm{~m}$

Vertical/surface libre haute mer

$16,54 \mathrm{~m}$

\subsection{3- Pieux}

La raideur des pieux s'élève à :

$\mathrm{K} 1=476 \mathrm{t} / \mathrm{m}$ au niveau basse mer

$\mathrm{K} 1=227 \mathrm{tm}$ au niveau haute mer 


\section{1. - Principe des essais sur houle sur modèle réduit}

Les essais consistaient à tester le comportement (mouvements) sur houle d'un modèle du ponton (ponton principal et ponton amovible) et à mesurer les efforts induits sur le système de pieux-guide, en tenant compte de la proximité de la berge. La Société CAREHNA a réalisé les essais dans la cuve à houle du Laboratoire National d'Hydraulique (LNH) d'EDF de Chatou (cf. figure 6).

La cuve a pour dimensions globales $33 \times 28 \times 1 \mathrm{~m}$. La cuve est équipée d'un générateur de houle de type "volet" de $17 \mathrm{~m}$ de long. Ce générateur est mobile sur un rail semi-circulaire dans un secteur de $180^{\circ}$. La gamme de fréquences de fonctionnement du batteur va de $0,4 \mathrm{Hg}$ à $2 \mathrm{Hg}$ (soit des périodes modèles de 0,55 à $2,5 \mathrm{~s}$.)

Etant donné les dimensions générales de l'installation et les caractéristiques-des agitations (périodes en particulier) à reproduire, l'échelle du modèle réduit a été fixée au $1 / 25 \mathrm{e}$.

\section{2 - Dispositif expérimental}

Etant donné le but recherché pour cette étude, il était indispensable de représenter, au moins schématiquement, la berge sur environ $200 \mathrm{~m}$ de part et d'autre du ponton flottant de manière à traiter les phénomènes de réflexion des vagues sur celle-ci.

La berge a été matérialisée sous forme d'un talus et d'un fond plat incliné dans la configuration basse mer. En configuration pleine mer, seul le talus était représenté.

Afin d'amortir la houle et d'adapter le coefficient de reflexion, le talus a été recouvert d'une couche de latex représentant les enrochements de la berge à pleine mer. En basse mer, les fonds ont été ramenés au plancher du bassin (cf. Figure 7).

Des pieux représentatifs du support de passerelle articulée et des ducs d'albe d'amarrage ont complété le modèle.

Les deux maquettes de pontons (ponton principal et ponton amovible) ont été réalisées à l'echelle $1 / 25$ du réel et permettent d'étudier alternativement les configurations "normale" et "rampe oblique". 
La liaison ponton-pieu a été modélisée en accord avec les degrés de liberté fixés pour l'installation.

La passerelle a été représentée par une poutre de manière à prendre en compte les effets d'inertie et de liaison. Elle est en appui glissant sur le ponton principal et articulée suivant un axe parallèle à la berge sur l'appui-fixe.

Les trois éléments du modèle, ponton principal, ponton amovible et passerelle ont été équilibrés indépendamment, en masses, centres de gravité et inerties.

Les pieux de guidage ont été modélisés pour respecter les raideurs de pieux au réel en tenant compte des débattements du ponton (notamment verticaux). Du fait de la souplesse des pieux, tous les mouvements du ponton par rapport à un repère fixe ont été autorisés.

En vue de simuler les conditions d'utilisation du ponton lors des phases chargement/déchargement, des essais ont été réalisés en configuration normale en présence d'un navire amarré.

\section{3 - Essais}

Les essais en houle régulière ont été réalisés par balayage en période (de 4 à 7 secondes), pour les deux incidences sélectionnées et pour une hauteur crête à creux de la houle incidente de l'ordre de 1,00 $\mathrm{m}$. Ces essais ont permis d'étudier le comportement du dispositif pour des houles dont la longueur d'onde était proche de la résonnance.

Les essais en houle aléatoire ont été, quant à eux, assurés pour deux spectres de houle de type Jonswap dont les caractéristiques étaient les suivantes:

$$
\begin{array}{ll}
\mathrm{H}_{\mathrm{s}}=1,00 \mathrm{~m} & \mathrm{~T}_{\text {pic }}=4 \mathrm{~s} \\
\mathrm{H}_{\mathrm{S}}=2,45 \mathrm{~m} & \mathrm{~T}_{\text {pic }}=5,8 \mathrm{~s}
\end{array}
$$

La durée de l'essai, de 450 secondes, correspondait à une durée de l'ordre de 40 minutes au réel. 


\section{4 - Résultatats des essais}

Les essais ont permis d'observer le comportement d"ensemble $\mathrm{du}$ ponton flottant et certains dispositifs ont fait l'objet d'une attention particulière en vue de valider ou adapter les choix techniques; ceci concerne :

- la mouille du pont,

- les risques de claque sous l'avant du ponton,

- la position de la passerelle par rapport à la houle.

L'analyse des mouvements indique la présence d'une résonance du dispositif pieux/ponton au voisinage des périodes de 6-7 secondes en configuration normale et à la période de 7 secondes en configuration rampe oblique.

Dans cette dernière configuration, les mouvements atteignent pour une incidence de $180^{\circ}, 1 \mathrm{~m} / \mathrm{m}$ de houle en cavalement $(\mathrm{x}), 0,65 \mathrm{~m} / \mathrm{m}$ de houle en embardée (y) et $0,4 \mathrm{~m} / \mathrm{m}$ de houle en pilonnement.

Cette résonance se répercute sur les efforts ponton/pieux qui augmentent fortement.

\section{a) Efforts sur les pieux}

Les efforts maximaux (crête-creux) mesurés sont de 300 tonnes sur le pieu avant (aval) et 270 tonnes sur le pieu arrière amont, ceci dans la configuration normale avec un Hmax de $3,90 \mathrm{~m}$, une période de pic de 5,8 secondes et une incidence de houle de $210^{\circ}$.

Les mouvements crête-creux maximaux associés sont:

$$
\begin{aligned}
& X_{g}=1.15 \mathrm{~m} \\
& Y_{g}=0,6 \mathrm{~m} \\
& \mathrm{Z}_{\mathrm{g}}=0,8 \mathrm{~m}
\end{aligned} \text { et } \quad \begin{aligned}
& \text { tangage }=3,6^{\circ} \\
& \text { roulis }=2^{\circ} \\
& \text { lacct }=2,6^{\circ}
\end{aligned}
$$

En configuration rampe oblique, les efforts les plus importants sont obtenus pour un Hmax de $3,20 \mathrm{~m}$, une incidence de $210^{\circ}$ et sont de 250 tonnes à l'avant et 200 tonnes à l'arrière. 


\section{b) Efforts de liaison}

Les efforts de liaison ont été mesurés dans la configuration rampe oblique entre le ponton principal et le ponton additionnel.

Les efforts maximaux crête-creux mesurés sont de l'ordre de:

$$
\begin{array}{ll}
\text { Fx }=50 \text { tonnes } ; & \mathrm{Mx}=1300 \text { tonnes*mètres } \\
. \mathrm{Fy}=120 \text { tonnes } ; & \mathrm{My}=400 \text { tonnes*mètres } \\
. \mathrm{Fz}=50 \text { tonnes } ; & \mathrm{Mz}=1200 \text { tonnes*mètres }
\end{array}
$$

\section{CONCLUSION}

Les essais physiques ont confirmé l'analyse des résultats de la modélisation mathématique en faisant notamment apparaitre une amplification des mouvements horizontaux et des efforts de liaison pontonpieux qui en découlent autour de $7 \mathrm{~s}$.

Toutefois l'analyse des divers mouvements horizontaux met en évidence des périodes propres comprises entre $3 \mathrm{~s}$ et $5 \mathrm{~s}$, et parfois multiples (cas des catamarans).

Le décalage entre période propre et résonance s'explique par le peu d'énergie des vagues courtes et la complexité du problème mécanique avec de forts couplages entre le cavalement, l'embardée et le lacet. 


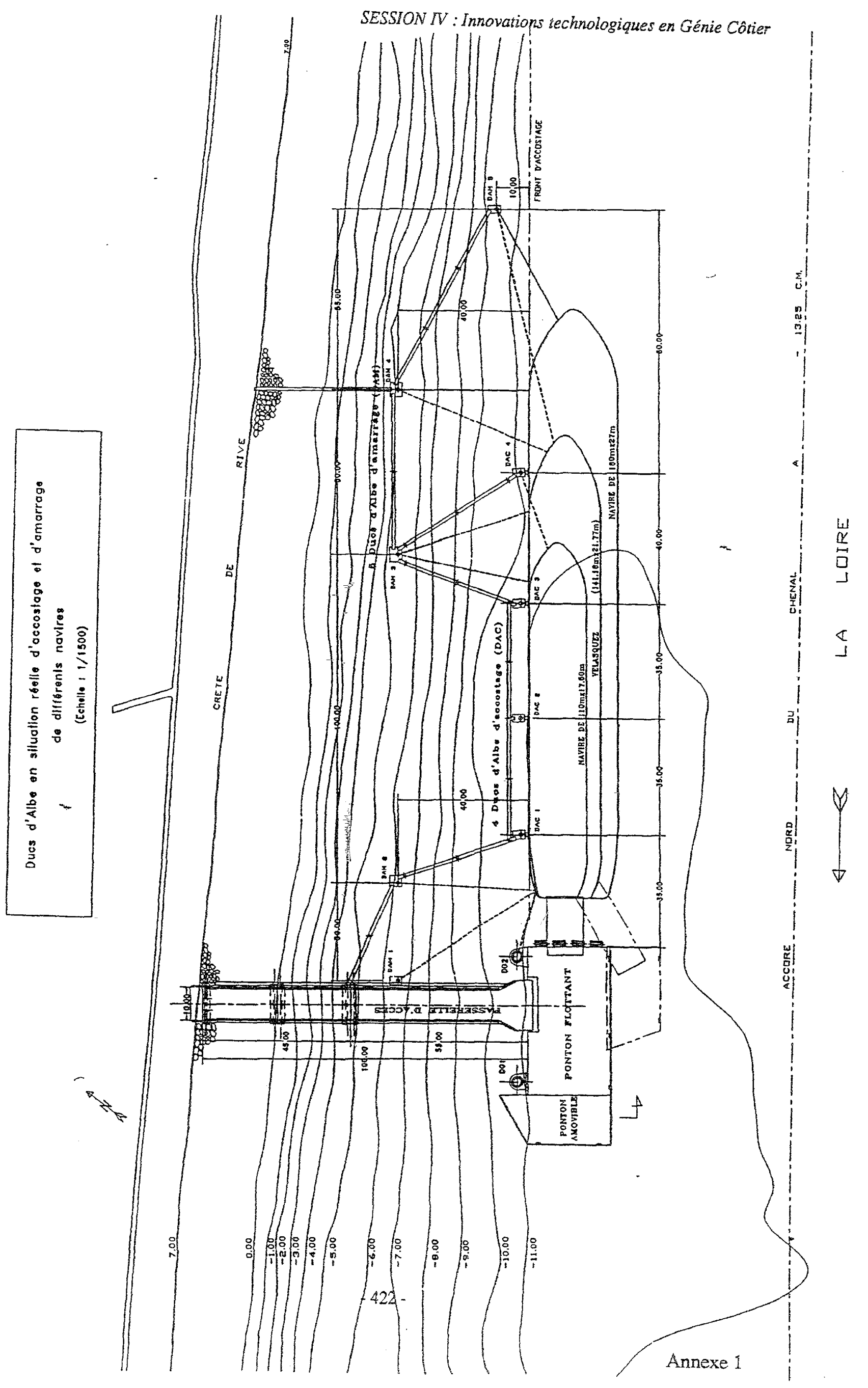



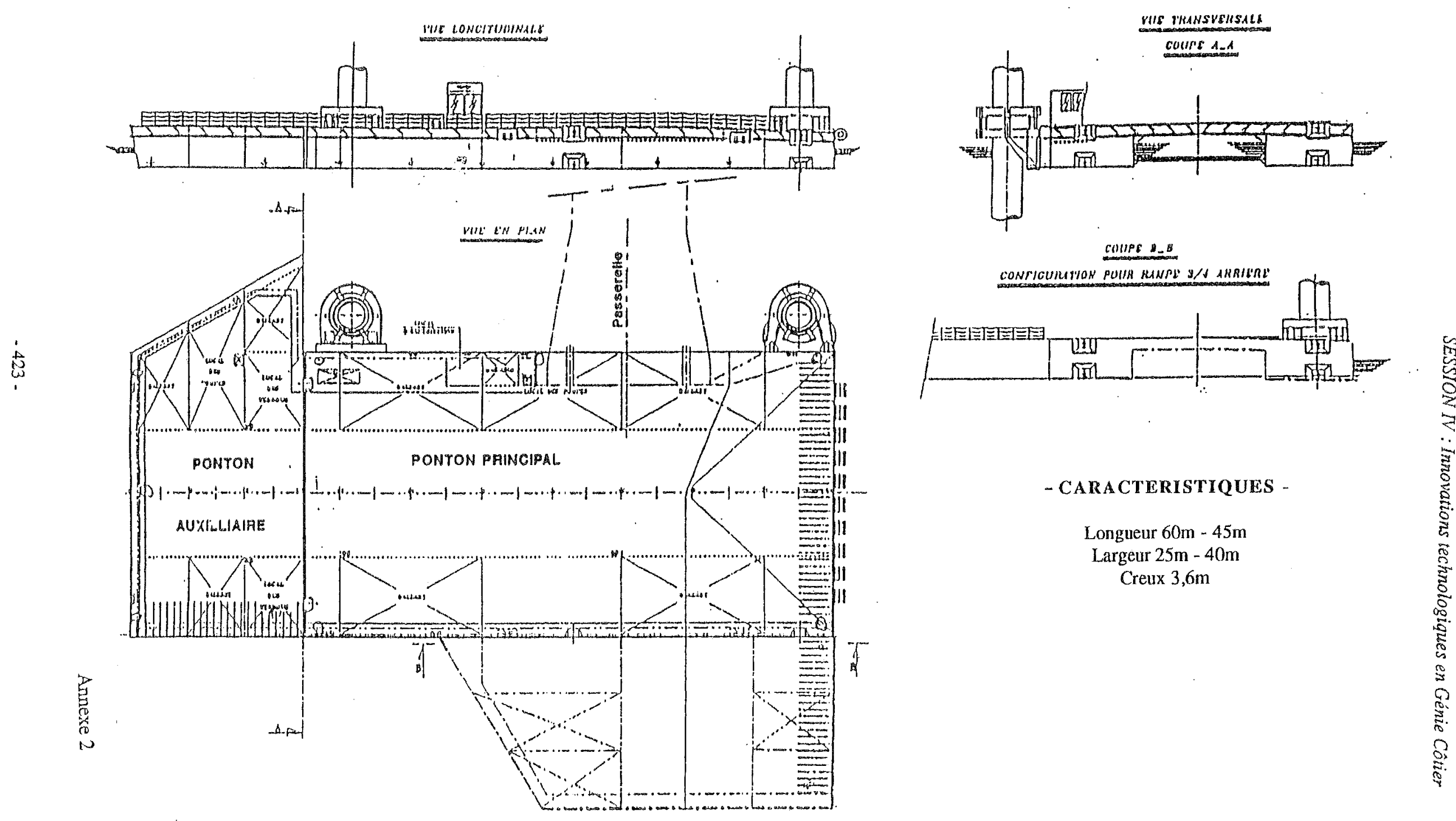


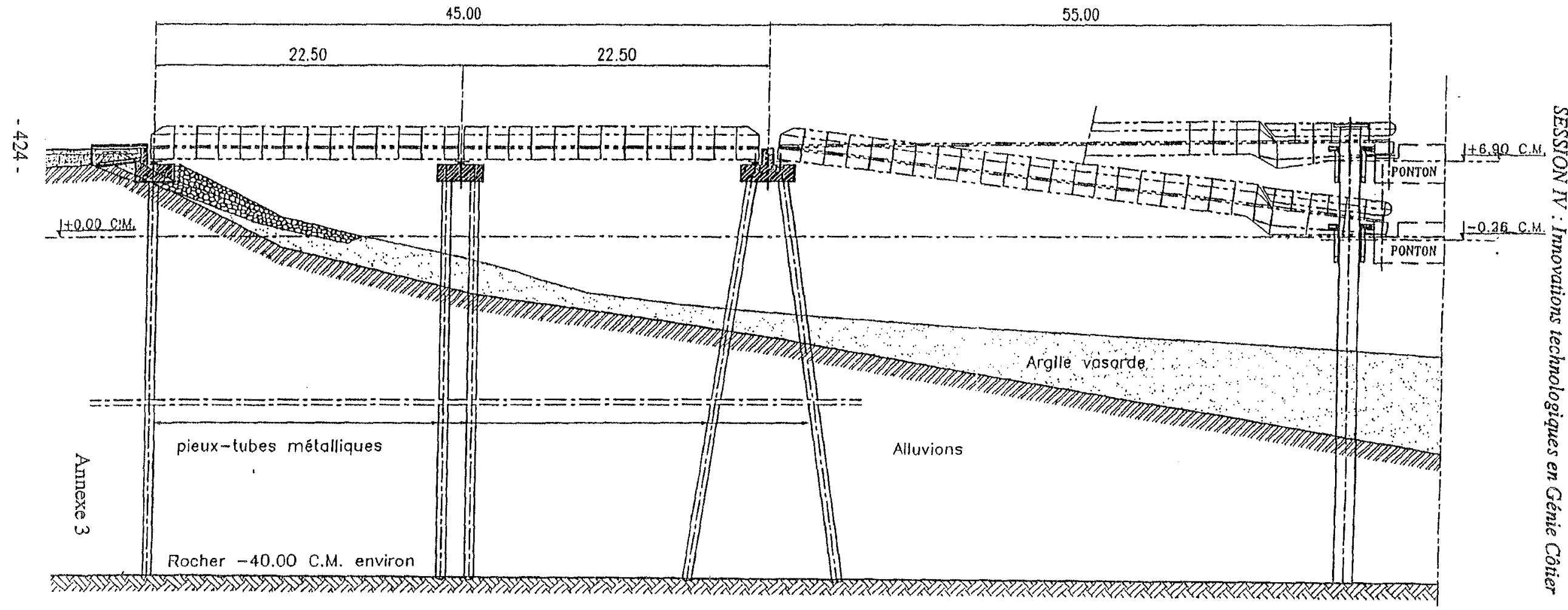




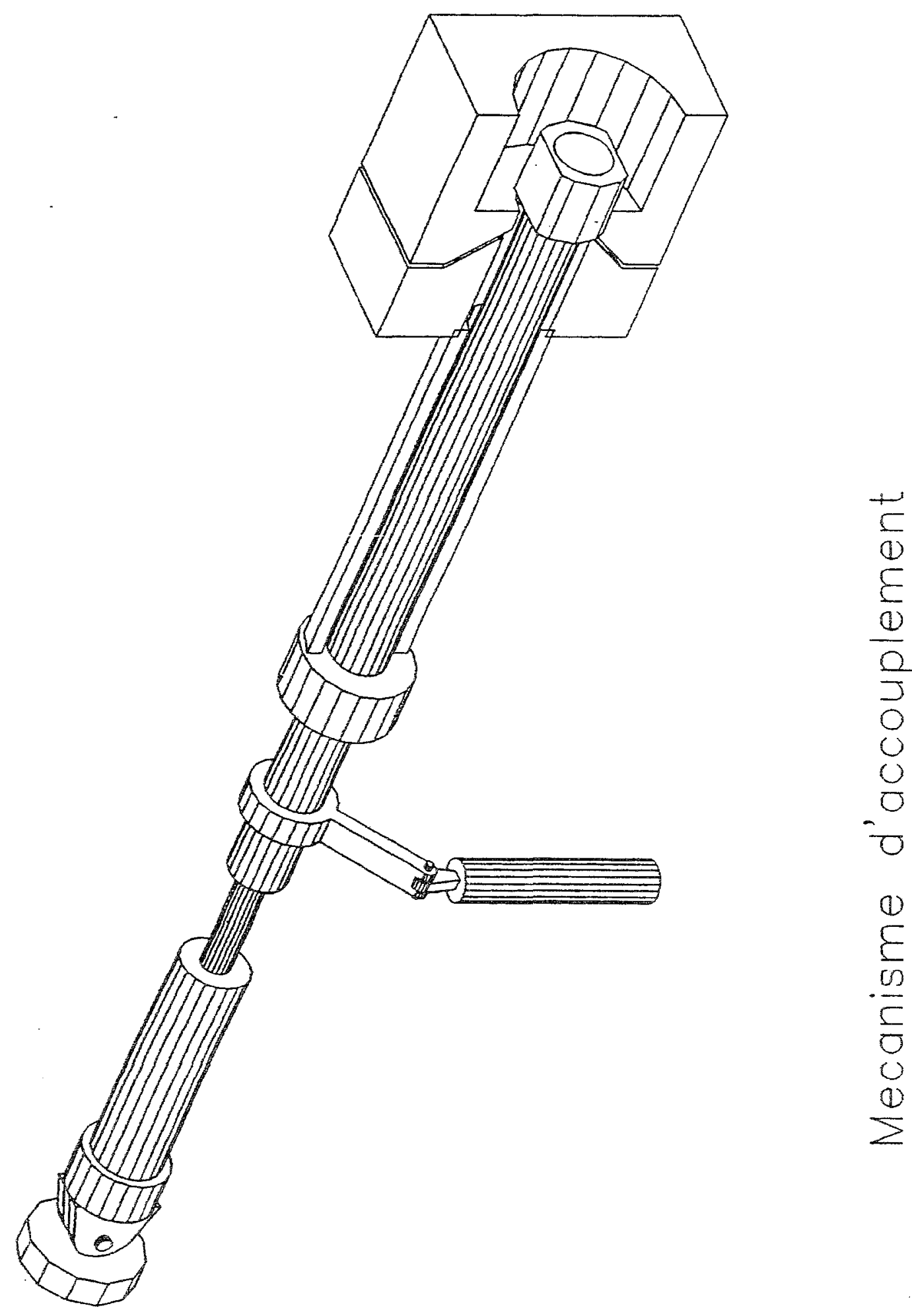


SESSION N : Innovations technologiques en Génie Côtier

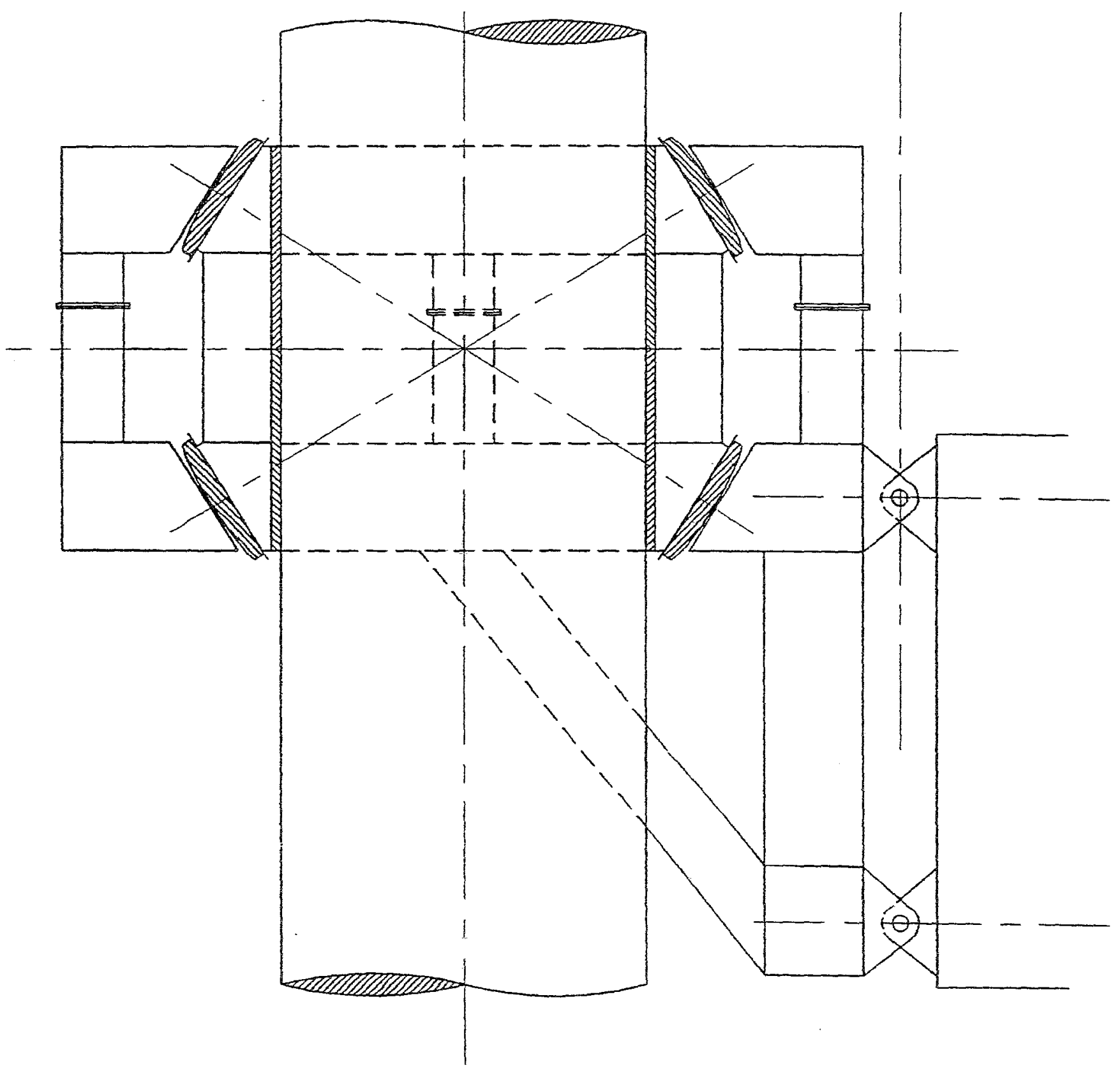

Collier à rotule

Principe

Annexe 5 
- Schéma des berges

Coupe longitudinale

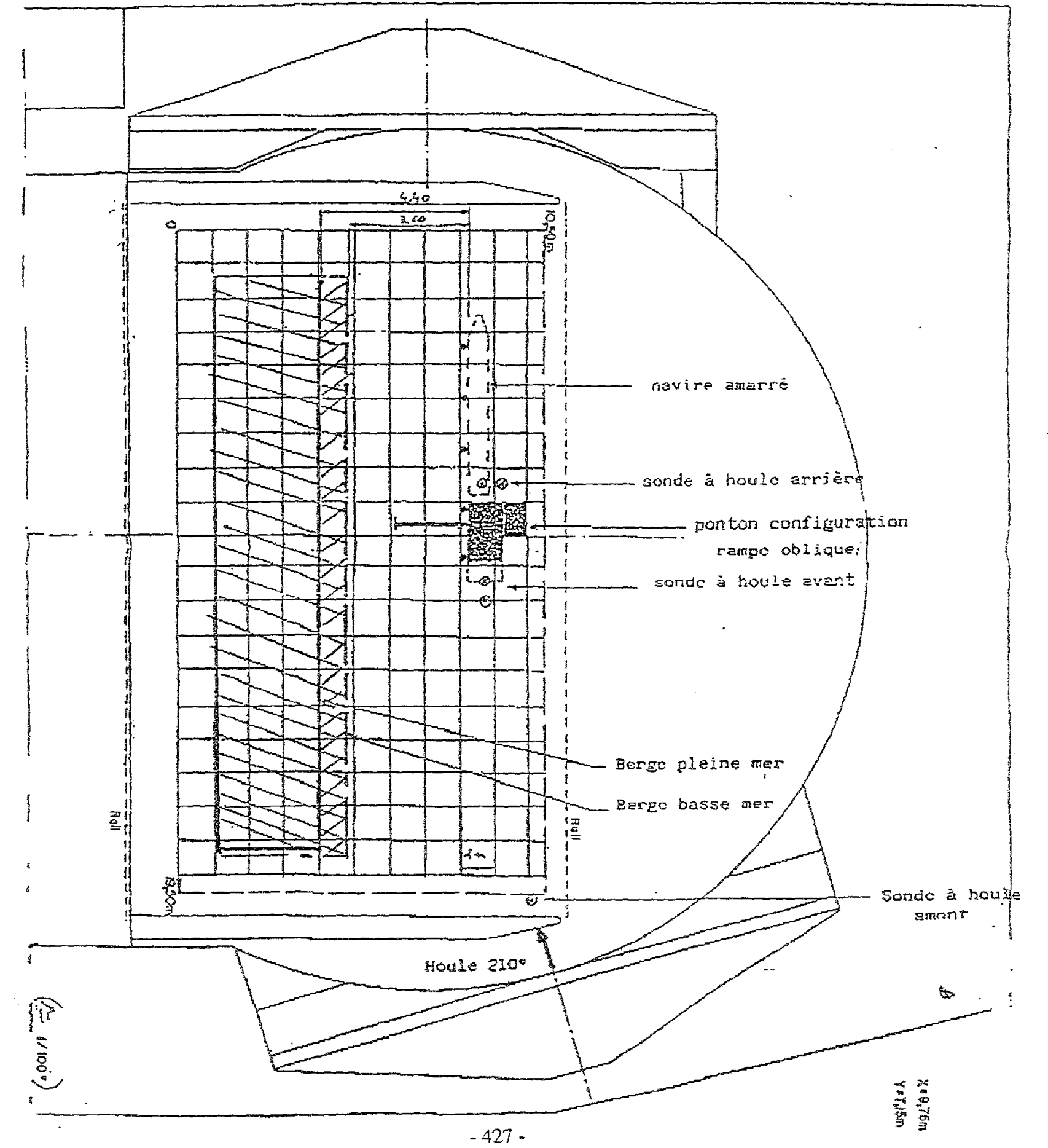

Arnexe 6 


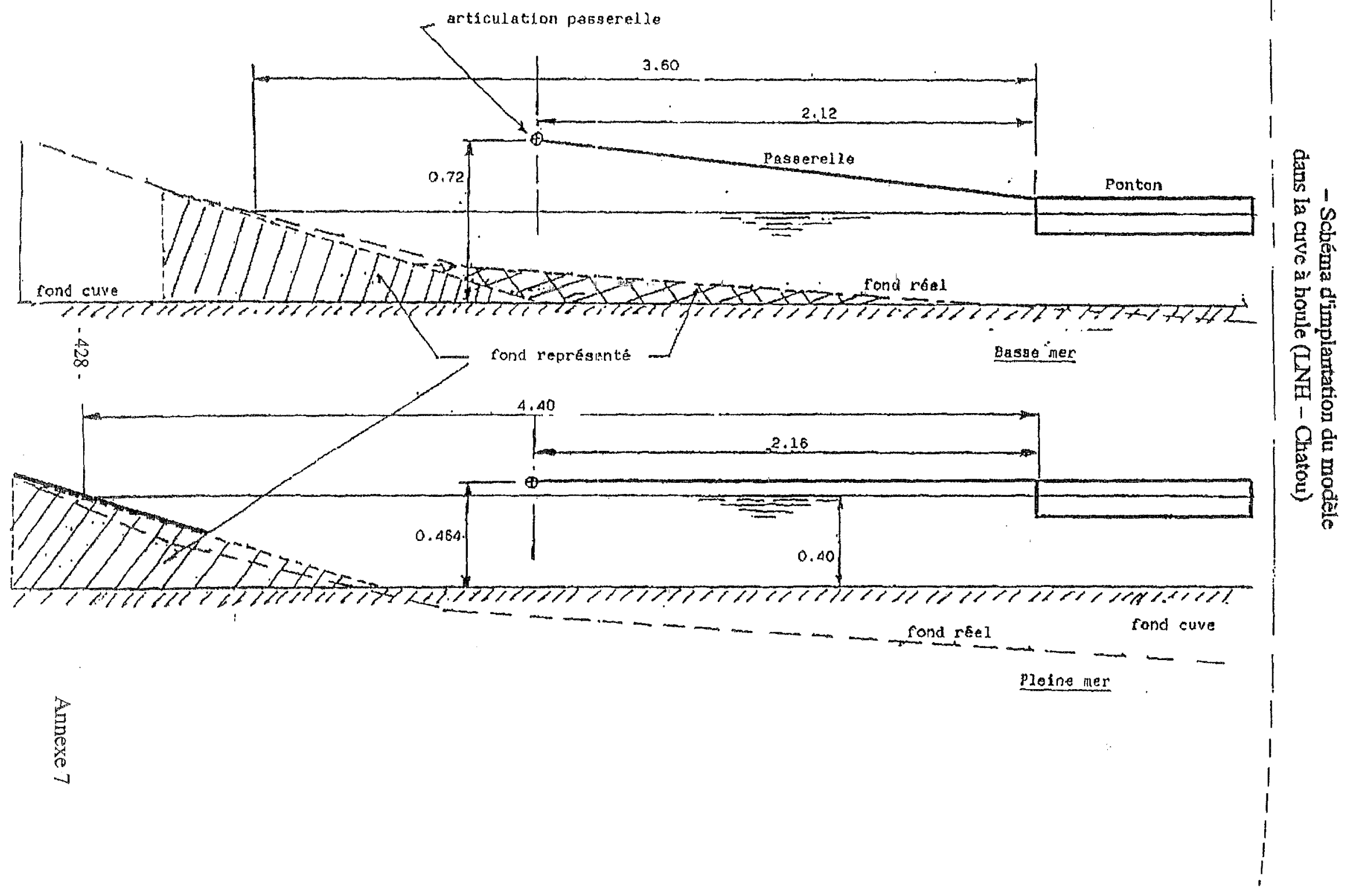

